\title{
Filter Banks for Hyperspectral Pixel Classification of Satellite Images
}

\author{
Olga Rajadell, Pedro García-Sevilla, and Filiberto Pla \\ Depto. Lenguajes y Sistemas Informáticos \\ Jaume I University, Campus Riu Sec s/n 12071 Castellón, Spain \\ \{orajadel,pgarcia,pla\}@lsi.uji.es \\ http://www.vision.uji.es
}

\begin{abstract}
Satellite hyperspectral imaging deals with heterogenous images containing different texture areas. Filter banks are frequently used to characterize textures in the image performing pixel classification. This filters are designed using different scales and orientations in order to cover all areas in the frequential domain. This work is aimed at studying the influence of the different scales used in the analysis, comparing texture analysis theory with hyperspectral imaging necessities. To pursue this, Gabor filters over complex planes and opponent features are taken into account and also compared in the feature extraction process.
\end{abstract}

\section{Introduction}

Nowadays imaging spectrometers are significantly increasing their spatial resolution. As their resolution increases, smaller areas are represented by each pixel in the images, encouraging the study of the relations of adjacent pixels (texture analysis) [9] [6]. However, not only the spatial resolution increases but also the spectral resolution. This entails dealing with a large number of spectral bands with highly correlated data [7].

Both dimensionality and texture analysis in hyperspectral imaginary have been tackled from different points of view in literature. Several solutions to the dimensionality problem can be found, such as selection methods based on mathematical dimensionality reduction [10] or methods based on information theory which try to maximize the information provided by different sets of spectral bands [7].

Moving to texture analysis, literature survey provides us with a wide variety of well known texture analysis methods based on filtering [8] [4]. It is well known that, when dealing with microtextures, the most discriminant information falls in medium and high frequencies [1] [9]. It has been recently proposed that spatial/texture analysis may significantly improve the results in pixel classification tasks for satellite images using a very reduced number of spectral bands [11]. Therefore, it may be convenient to identify the influence of each frequency band separately in order to compare the textural information with the specific necessities of hyperspectral satellite imaging.

Besides, color opponent features were first introduced in color texture characterization with fairly good performance [3] and later extended to deal with multi-band texture images [4]. However, they have never been used to perform pixel classification tasks in satellite images. In this paper, we study several Gabor filter banks as well as multi-band opponent features for pixel classification tasks.

E. Bayro-Corrochano and J.-O. Eklundh (Eds.): CIARP 2009, LNCS 5856, pp. 1039-1046, 2009.

(C) Springer-Verlag Berlin Heidelberg 2009 


\section{Filter Banks and Feature Extraction}

Applying a filter over an image band provides a response for each pixel. If a filter bank is applied, a pixel can be characterized by means of the responses generated by all filters. It is possible to apply a filter in the space domain by a convolution or in the frequency domain by a product. In both cases, the response is the corresponding part of the original pixel value which responds to the filter applied [12].

When using filter banks, they are generally designed considering a dyadic tessellation of the frequency domain, that is, each frequency band (scale) considered is double the size of the previous one. It should not be ignored that this tessellation of the frequency domain thoroughly analyzes low frequencies giving less importance to medium and higher frequencies. Because the purpose of this work is to study the importance of texture in the pixel classification task, an alternative constant tessellation (given the same width to all frequency bands) is proposed in order to ensure an equal analysis of all frequencies.

\subsection{Gabor Filters}

Gabor filters consist essentially of sine and cosine functions modulated by a Gaussian envelope that achieve optimal joint localization in space and frequency. They can be defined by eq. (1) and (2) where $m$ is the index for the scale, $n$ for the orientation and $u_{m}$ is the central frequency of the scale.

$$
\begin{aligned}
& f_{m n}^{r e a l}(x, y)=\frac{1}{2 \pi \sigma_{m}^{2}} \exp \left\{-\frac{x^{2}+y^{2}}{2 \sigma_{m}^{2}}\right\} \times \cos \left(2 \pi\left(u_{m} x \cos \theta_{n}+u_{m} y \sin \theta_{n}\right)\right) \\
& f_{m n}^{\text {imag }}(x, y)=\frac{1}{2 \pi \sigma_{m}^{2}} \exp \left\{-\frac{x^{2}+y^{2}}{2 \sigma_{m}^{2}}\right\} \times \sin \left(2 \pi\left(u_{m} x \cos \theta_{n}+u_{m} y \sin \theta_{n}\right)\right)
\end{aligned}
$$

If symmetrical filters are considered only the real part must be taken into account.

\subsection{Gabor Filters over Complex Planes}

Texture analysis in multi-channel images has been generally faced as a multi-dimensional extension of techniques designed for mono-channel images. In this way, images are decomposed into separated channels and the same feature extraction process is performed over each channel. This fails in capturing the interchannel properties of a multi-channel image.

To describe the inter-channel properties of textures we propose features obtained using Gabor filters over complex planes. This means that instead of using each spectral band individually, we take advantage of the complex definition and introduce the data of two spectral bands into one complex band, one as the real part and the other one as the imaginary part. In this way we involve pairs of bands in each characterization process, as it happens for the opponent features. As a result, for a cluster of spectral bands, we will consider all possible complex bands (pairs of bands). The Gabor filter bank will be applied over all complex bands as shown in eq. 3, where $I^{i}(x, y)$ is the $i^{t h}$ spectral band.

$$
h_{m n}^{i j}(x, y)=\left(I^{i}(x, y)+I^{j}(x, y) i\right) * f_{m n}(x, y)
$$


The feature vector for each pixel in the image is composed of the response for that pixel to all filters in the filter bank, that is:

$$
\psi_{x, y}=\left\{h_{m n}^{i j}(x, y)\right\}_{\forall i, j / i \neq j, \forall m, n}
$$

The size of the feature vector varies with the number of complex bands. For each complex band, one feature is obtained for each filter applied what means that there will be as many features as filters for each complex band and as many complex bands as combinations without order nor repetition may be done with two bands in the cluster $B$. The total number of features is given by eq. 5 where $M$ stands for the number of scales and $N$ for the number of orientations.

$$
\operatorname{size}\left(\psi_{x, y}\right)=M \times N \times\left(\begin{array}{l}
B \\
2
\end{array}\right)
$$

\subsection{Opponent Features}

Opponent features combine spatial information across spectral bands at different scales and are related to processes in human vision [3]. They are computed from Gabor filters as the difference of outputs of two different filters. The combination among filters are made for all pair of spectral bands $i, j$ with $i \neq j$ and $\left|m-m^{\prime}\right| \leq 1$ :

$$
d_{m m^{\prime} n}^{i j}(x, y)=h_{m n}^{i}(x, y)-h_{m^{\prime} n}^{j}(x, y)
$$

In this case, the feature vector for a pixel is the set of all opponent features for all spectral bands.

$$
\varphi_{x, y}=\left\{d_{m m^{\prime} n}^{i j}(x, y)\right\}_{\forall i, j / i \neq j, \forall m, m^{\prime} /\left|m-m^{\prime}\right| \leq 1, \forall n}
$$

Hence, the size of the opponent feature vector also depends on the number of bands, scales, and orientations:

$$
\begin{array}{r}
\operatorname{size}\left(\varphi_{x, y}\right)=\left(\left(\begin{array}{c}
B \\
2
\end{array}\right) \times M+B^{2} \times(M-1)\right) \times N= \\
=\operatorname{size}\left(\psi_{x, y}\right)+B \times(B-1) \times(M-1) \times N
\end{array}
$$

Note that, in this case, the number of features is considerably increased.

\section{Experimental Setup}

The hyperspectral image database 92AV3C image has been used in the pixel classification experiments. It was provided by the Airborne Visible Infrared Imaging Spectrometer (AVIRIS) [13]. The 20-m GSD data was acquired over the Indian Pine Test Site in Northwestern Indiana in 1992. From the original 220 AVIRIS spectral bands our band selection method provides us with ten clusters of bands which are sets of bands that are intended to maximize the information provided [7]. The first cluster contains just one bands, the second contains two bands, and so on. 
The experimental activity was held using two filter banks. For the first one, six dyadic scales (the maximum starting from width one and covering all the image) and four orientations were used. For the second one, eight constant frequency bands and four orientations were considered. It has been introduced certain degree of overlapping as recommended in [2]. Gaussian distributions are designed to overlap each other when achieving a value of 0.5 .

For each of the scales a classification experiment was held using only the features provided for that scale. In addition, an analysis of the combination of adjacent scales have been performed. In order to study the importance of low frequencies an ascendent joining was performed, characterizing pixels with the data provided by joined ascendent scales. Similarly, the study of the high frequencies was carried out by a descendant joining. Also for medium frequencies, central scales are considered initially and adjacent lower and higher scales are joined gradually.

The pixels in the image database are divided in twenty non overlapping sets keeping the a priori probability of each class. Therefore, no redundancies are introduced and each set is a representative set of the bigger original one. Ten classification attempts were carried out for each experiment with the k-nearest neighbor algorithm with $k=3$ and the mean of the error rates of these attempts was taken as the final performance of the classifier. Each classification attempt uses one of these sets for training and another as test set. Therefore, each set was never used twice in the same experiment.

\section{Evaluation of the Results}

Figure 1 shows the percentages of correct pixel classification obtained for the experiments that used the dyadic filter bank. Figure 2 shows similar results when the constant filter bank was used.

As it can be observed from both figures, when the characterization processes included all scales, the filter bank using the dyadic tessellation outperforms the constant one. It seems clear that the better the low frequencies are analyzed the better the pixels are characterized. This means that, for this sort of images, the texture information, although still helps in the characterization process, is significantly lower than the information contained in the low frequencies. It can be seen that no scale can ever outperform the classification rates achieved by scale one which achieve up to $81 \%$ by itself. In general, the more detail is obtained from low frequencies the best the image is characterized.

For the dyadic tessellation, although scales two and three do not outperform scale one when characterizing independently (Fig. 1 $\mathrm{a}-\mathrm{b}$ ), their performance is considerably high. Because the first scales cover a very small part of the frequency domain, the characterization joining scales 1, 2 and 3 improve the pixel classification rates (Fig. 1 d). In a nutshell, when all (six) scales are used, the classification rates are better than the ones obtained using just the first scale. However, it is worse than the results obtained for the first three scales although having a double number of features. The descendent and central joinings (Figs. 1, $\mathrm{f}$ and $1 \mathrm{~g}$-h) clearly show that the performance increases significantly as features derived from lower frequencies are considered. 


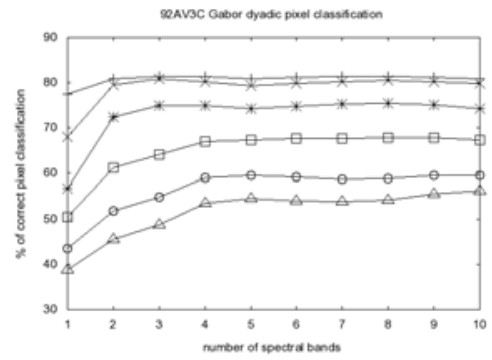

(a)

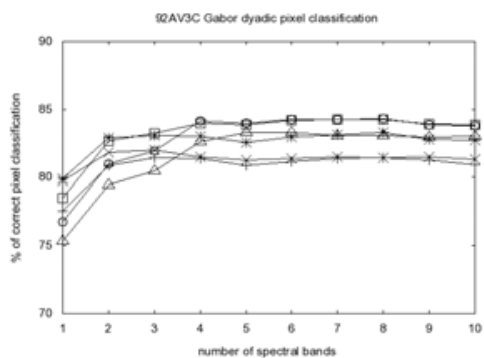

(c)

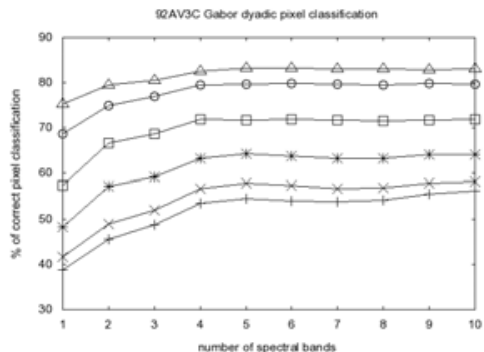

(e)

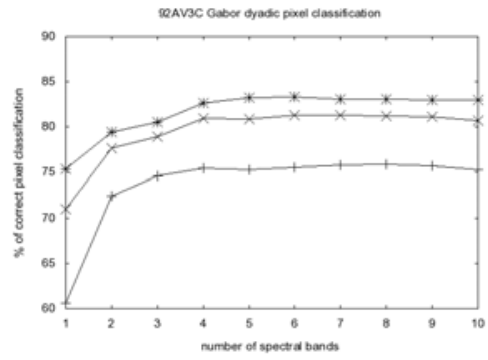

(g)

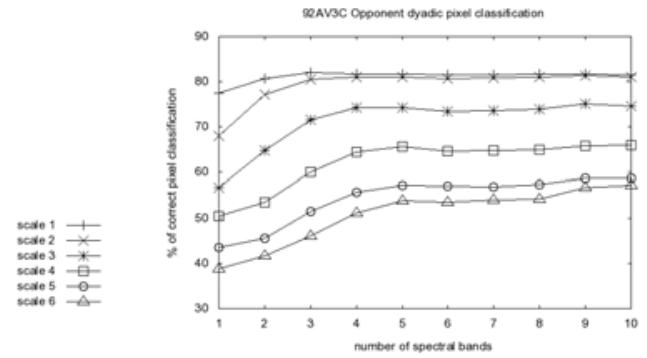

(b)

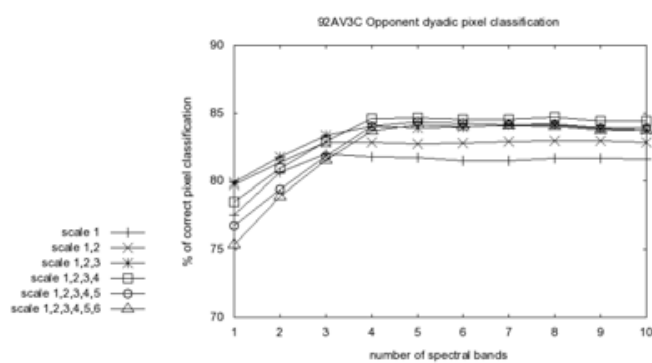

(d)

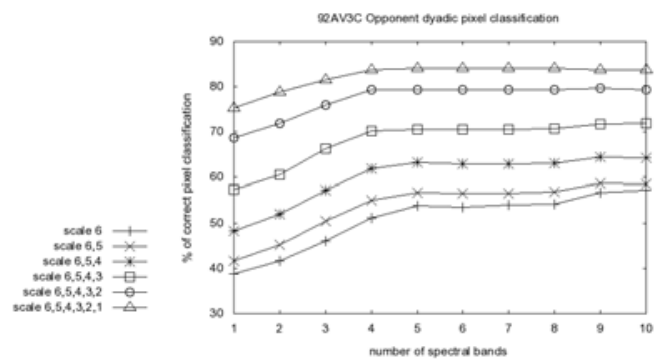

(f)

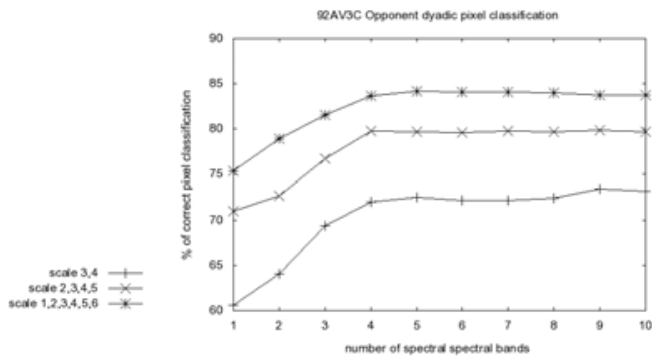

(h)

Fig. 1. Pixel classification rates using the filter bank with dyadic tessellation. (a,c,e,g) Gabor features over complex planes (b,d,f,h) Opponent features (a,b) Individual scales (c,d) Ascendent join $(\mathrm{e}, \mathrm{f})$ Descendent join $(\mathrm{g}, \mathrm{h})$ Central join. Note the different ranges over the Y-axis in each graph. 


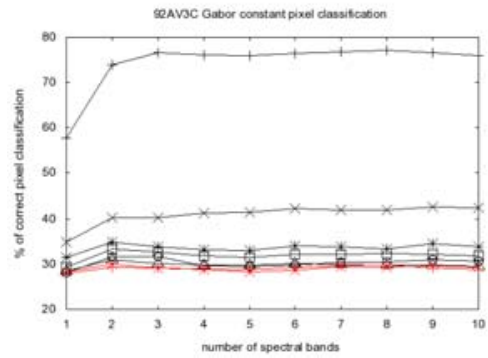

(a)

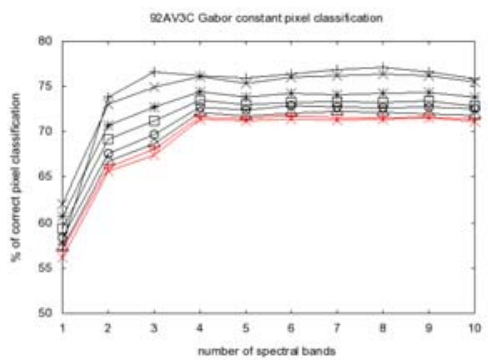

(c)

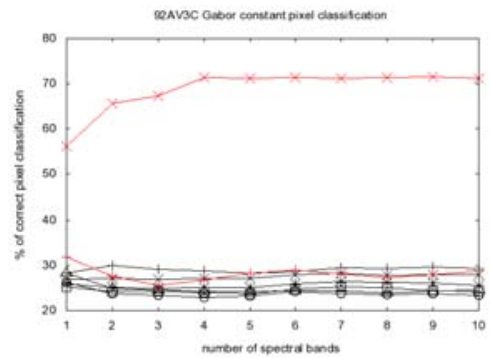

(e)

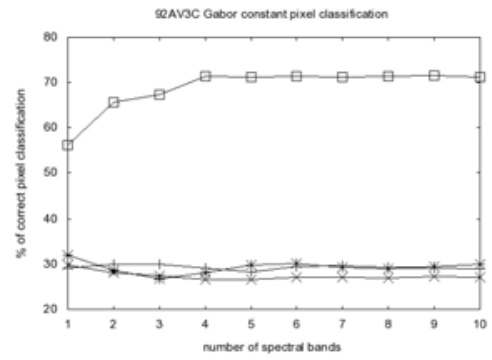

(g)

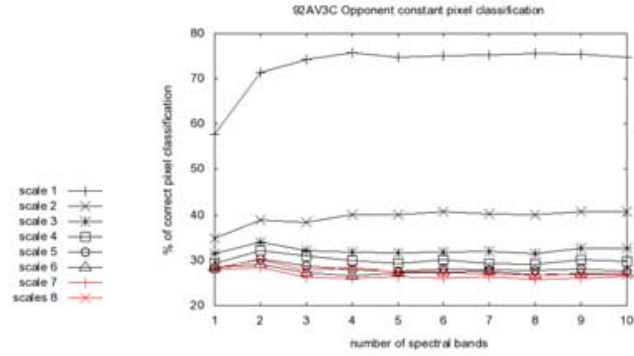

(b)

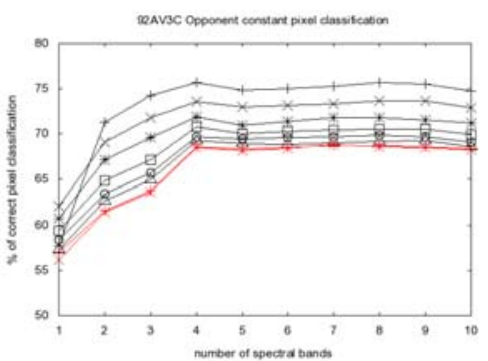

(d)

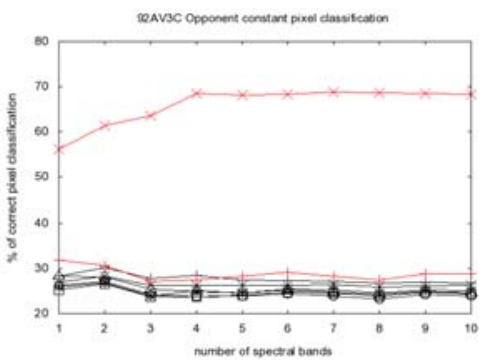

(f)

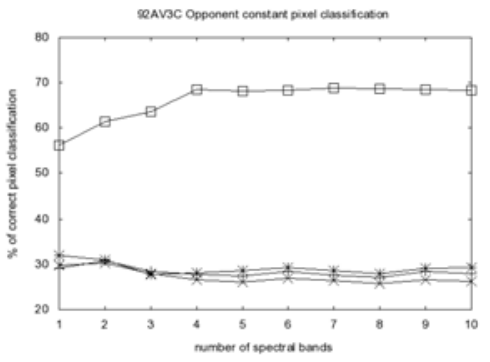

(h)

Fig. 2. Pixel classification rates using the filter bank with constant tessellation. (a,c,e,g) Gabor features over complex planes (b,d,f,h) Opponent features (a,b) Individual scales (c,d) Ascendent join $(\mathrm{e}, \mathrm{f})$ Descendent join $(\mathrm{g}, \mathrm{h})$ Central join. Note the different ranges over the Y-axis in each graph. 
Regarding the filter bank, using a constant tessellation (Fig. 2), the first scale is the only one containing discriminant information. This first scale is wide enough in this case to include the information of several scales of the dyadic tessellation. It is very clear from the graphs that the features derived from other scales do not help the characterization processes as the classification rates always decrease. It can be noticed that the best classification rates obtained for the dyadic tessellation is over $84 \%$ but is only about $77 \%$ for the constant tessellation.

Last but not least, the comparison between the feature extraction methods suggest that opponent features perform similarly to Gabor filters over complex planes. It seems that Gabor features provide better results when using a very small number of spectral bands whereas opponent features provide slightly higher classification rates when more spectral bands are used. Nevertheless, on the whole, the characterization with opponent features requires a larger number of features than Gabor filters, which may worsen performance if a large number of spectral bands is to be considered.

Briefly, spatial analysis between pixels improves hyperspectral satellite images characterization [11] but the nature of this kind of images, which are heterogeneous due to being composed of different homogeneous areas, made low frequencies very important for the characterization task, while texture information may help the process, but not significantly. Furthermore, including much more information but the provided by the low frequency analysis may even decrease the performance because of the so call Hughes phenomenon [5].

\section{Conclusions}

An analysis of the contribution of each scale to the characterization of hyperspectral images has been performed. As it is known in the texture analysis field, medium and high frequencies play an essential role in texture characterization. However, satellite images cannot be considered as pure texture images since the homogeneity of the different areas in the image is more important than the texture these areas may content. A thoroughly analysis of the contribution of each independent scale and the group composed by low, medium or high frequencies has been carried out. It has been shown that a detailed analysis of low frequencies helps the characterization improving performance. Also a few scales could be considered in the feature extraction process providing by themselves very high classification rates with a few number of features. The comparison between Gabor filters over complex plains and opponent features has shown that the classification rates obtained are almost identical in both cases. The main difference is the number of features required in each case, being much larger for the opponent features.

\section{Acknowledgment}

This work has been partly supported by Fundació Caixa Castelló-Bancaixa through grant FPI PREDOC/2007/20 and project P1-1B2007-48, project CSD2007 00018 from Consolider Ingenio 2010, and project AYA2008-05965-C04-04 from Spanish CICYT. 


\section{References}

1. Chang, T., Kuo, C.C.J.: Texture analysis and classification with tree-structured wavelet transform. IEEE Trans. on Geoscience \& Remote Sensing 2, 429-441 (1993)

2. Bianconi, F., Fernández, A.: Evaluation of the effects of Gabor filter parametres on texture classification. Patt. Recogn. 40, 3325-3335 (2007)

3. Jaim, A., Healey, G.: A multiscale representation including oppponent color features for texture recognition. IEEE Trans. Image Process. 7, 124-128 (1998)

4. Shi, M., Healey, G.: Hyperspectral texture recognition using a multiscale opponent representation. IEEE Trans. Geoscience and remote sensing 41, 1090-1095 (2003)

5. Hughes, G.F.: On the mean accuracy of statistical pattern recognizers. IEEE Trans. Inf. Theory $14,55-63(1968)$

6. Landgrebe, D.A.: Signal Theory Methods in Multispectral Remote Sensing. Wiley, Hoboken (2003)

7. Martínez-Usó, A., Pla, F., Sotoca, J.M., García-Sevilla, P.: Clustering-based Hyperspectral Band selection using Information Measures. IEEE Trans. on Geoscience \& Remote Sensing 45(12), 4158-4171 (2007)

8. Mercier, G., Lennon, M.: On the characterization of hyperspectral texture. IEEE Trans. Inf. Theory 14, 2584-2586 (2002)

9. Petrou, M., García-Sevilla, P.: Image Processing: Dealing with Texture. John Wiley and Sons, Chichester (2006)

10. Plaza, A., Martinez, P., Plaza, J., Perez, R.: Dimensionality reduction and classification of hyperspectral image data using sequences of extended morphological transformations. IEEE Trans. Geoscience and remote sensing 43(3), 466-479 (2005)

11. Rajadell, O., García-Sevilla, P., Pla, F.: Textural features for hyperspectral pixel classification. In: Araujo, H., et al. (eds.) IbPRIA 2009. LNCS, vol. 5524, pp. 497-504. Springer, Heidelberg (2009)

12. Shaw, G., Manolakis, D.: Signal processing for hyperspectral image explotation. IEEE Signal Process. Mag. 19(1), 12 (2002)

13. Vane, G., Green, R., Chrien, T., Enmark, H., Hansen, E., Porter, W.: The Airborne Visible Infrared Imaging Spectrometer Remote Sens. Environ. 44, 127-143 (1993) 